\title{
ASPECTOS TAXONÔMICOS DE TRÊS ESPÉCIES DE CORALINÁCEAS NÃO GeNiculadas DO Litoral do ESTADO DA BAHIA, BRASIL
}

\author{
José Marcos de Castro Nunes ${ }^{1,2,5}$, Silvia Maria Pita de Beauclair Guimarães ${ }^{3}$, \\ Alejandro Donnangelo ${ }^{4}$, Julyana Farias ${ }^{4} \&$ Paulo Antunes Horta ${ }^{4}$
}

\begin{abstract}
RESUMO
(Aspectos taxonômicos de três espécies de coralináceas não geniculadas do litoral do estado da Bahia, Brasil) As algas calcárias não articuladas, representadas pela ordem Corallinales são amplamente distribuídas pelos oceanos, ocorrem desde a zona das marés até regiões profundas e apresentam extrema importância ecológica e econômica. Apesar destes aspectos, as algas calcárias não articuladas ainda são pouco conhecidas no Brasil. Com o intuito de contribuir para o conhecimento taxonômico desse grupo, neste trabalho são apresentadas descrições de três espécies algas de calcárias não articuladas encontradas no litoral do estado da Bahia: Sporolithon episporum, Mesophyllum erubescens e Lithophyllum stictaeforme. Palavras-chave: algas calcárias, Corallinales, Sporolithon, Mesophyllum, Lithophyllum.
\end{abstract}

\section{Abstract}

(Taxonomic aspects of three species of non geniculate coralline algae from Bahia State, Brazil) The non geniculated coralline algae, represented by the order Corallinales are largely distributed in all seas, occur from the tidal zone down to deep regions and present highly important ecological and economical role. Although these, non geniculated coralline algae are poorly known in Brazil. In order to contribute to the taxonomic knowledge of these algae group, in this work it was presented descriptions of three species from Bahia State coast: Sporolithon episporum, Mesophyllum erubescens and Lithophyllum stictaeforme.

Key words: coralline algae, Corallinales, Sporolithon, Mesophyllum, Lithophyllum.

\section{INTRODUÇ̃̃o}

As algas coralináceas não articuladas ou incrustantes são encontradas na maioria dos habitats marinhos, desde zonas tropicais até regiões polares ocorrendo desde a zona das marés até profundidades de 268 metros (Littler et al. 1985). São elementos importantes para a construção de formações recifais e de bancos de rodolitos, onde são os principais organismos formadores (Foster et al. 2007). Apesar de apresentarem elevada importância ecológica e econômica, as algas calcárias não articuladas, ou calcárias incrustantes (Corallinales) são pobremente conhecidas no mundo, e especialmente no Brasil (Horta 2002).
Segundo Harvey et al. (2003), a ordem Corallinales é dividida em três famílias, Hapalidiaceae, Corallinaceae e Sporolithaceae. Esta última foi estabelecida por Verheij (1993) por apresentar tetrasporângios cruciados, e está representada pelos gêneros Sporolithon e Heydrichia (Towsend \& Borowitzka 2001). As Corallinaceae, representadas por gêneros como Lithophyllum e Titanoderma, apresentam tetrasporângios zonados em conceptáculos uniporados sem tampão apical, enquanto que as Hapalidiaceae, representadas por gêneros como Lithothamnion e Phymatolithon, apresentam conceptáculos tetrasporangiais multiporados entre outras características morfoanatômicas e moleculares (Harvey et al. 2003).

\footnotetext{
Artigo recebido em 04/2007. Aceito para publicação em 01/2008.

${ }^{1}$ Laboratório de Algas Marinhas - LAMAR, Departamento de Botânica, Instituto de Biologia - UFBA, Campus de Ondina, 40170-280, Salvador, BA, Brasil.

${ }^{2}$ Departamento de Ciências Exatas e da Terra, Campus II - Alagoinhas, Universidade do Estado da Bahia, Rodovia Alagoinhas-Salvador, km 03, 48000-000, Alagoinhas, BA, Brasil.

${ }^{3}$ Seção de Ficologia, Instituto de Botânica de São Paulo, Av. Miguel Stéfano, 3684, 01061-970, São Paulo, SP, Brasil. ${ }^{4}$ Laboratório de Ficologia, Departamento de Botânica, Centro de Ciências Biológicas, Universidade Federal de Santa Catarina, 88010-970, Florianópolis, SC, Brasil.

${ }^{5}$ Autor para correspondência: jmcnunes@ufba.br
} 
No Brasil, até meados da década de 70, os estudos sobre algas calcárias incrustantes se limitaram a algumas citações de espécies (Martens 1870; Taylor 1931; Oliveira Filho et al. 1979). O trabalho de Tomita (1976), que apresenta uma análise detalhada do gênero Sporolithon no litoral brasileiro, marcou o início de um novo período. No final do século XX e início do século XXI os estudos mais detalhados sobre o grupo se intensificam. Horta (2000) ao estudar as algas marinhas do infralitoral das Regiões Sul e Sudeste do Brasil, descreve oito espécies de coralináceas não articuladas. Horta (2002) apresenta levantamento histórico sobre os estudos taxonômicos em coralináceas não articuladas realizados no Brasil. Tâmega \& Figueiredo (2005) estudaram a distribuição de algas calcárias incrustantes em diferentes ambientes no litoral do Rio de Janeiro identificando a espécie Hydrolithon samoënse (Foslie) D. Keats \& Y. Chamberlain. Amado Filho et al. (2007) citam cinco gêneros como formadores de rodolitos para o sul do Espírito Santo. No litoral baiano, mais precisamente, em Abrolhos, Figueiredo (1997) e Figueiredo \& Steneck (2002) enfocam o grupo das coralináceas incrustantes, sendo que em ambos os trabalhos são abordados aspectos florísticos e ecológicos, onde foram citadas as espécies: Hydrolithon boergesenii (Foslie) Foslie, Neogoniolithon accretum, (Foslie \& M. Howe) Setch. \& L. R.Mason., Porolithon pachydermum (Foslie) Foslie, Lithophyllum congestum (Foslie) Foslie, L. frondosum (L. Dufour) G. Furnari, Cormaci \& Alongi (como Titanoderma bermudense), Lithothamnion occidentale (Foslie) Foslie, L. sejunctum Foslie, Mesophyllum erubescens (Foslie) Lemoine, Sporolithon dimotum (Foslie \& M. Howe) Yam.-Tomita ex M.J. Wynne e $S$. episporum (M. Howe) E. Y. Dawson.

Com o intuito de contribuir para o conhecimento taxonômico desse grupo no Brasil, no presente trabalho são apresentadas descrições de três espécies de algas calcárias não articuladas do litoral baiano pertencentes aos gêneros Sporolithon, Mesophyllum e Lithophyllum.

\section{Material e Métodos}

A metodologia utilizada é resultado da adaptação de métodos já propostos por Woelkerling (1988), Riosmena-Rodriguez (1993) e Moura et al. (1997). Todo material foi fixado em formol $4 \%$ em água do mar. Após 24 horas o material foi transferido para uma solução de etanol a $70 \%$ e glicerol a $10 \%$.

Para a confecção de cortes histológicos o material foi descalcificado em ácido nítrico 0,6 M e desidratado em série alcoólica de 30, $50,70,90$ e $100 \%$ de etanol, a intervalos de 20 minutos. Em seguida o material foi infiltrado por pelo menos 12 horas em solução de metacrilatoglicol ("Historesin embedding Kit" Leica, marca Reichert-Jung) e emblocado segundo as instruções fornecidas pelo referido fabricante. Para a confecção dos cortes histológicos de 3-15 mm de espessura, foi utilizado micrótomo rotativo Leica, modelo 2040, e para a montagem das lâminas seguiu-se à metodologia proposta por Moura et al. (1997).

Para as observações por microscopia eletrônica de varredura, o material armazenado em álcool e glicerol, foi transferido para uma solução de álcool $70 \%$ e após 12 horas seco por cerca de 48 horas em estufa a aproximadamente $50^{\circ} \mathrm{C}$. O material fértil foi fraturado e devidamente posicionado em suporte com o auxílio de fitas adesivas dupla face e metalizado em ouro, com um tempo de exposição de 90 segundos, a uma corrente de 50 mA. Foi utilizado o microscópio eletrônico de varredura Hitachi X650, equipado com uma câmera Mamiya $6 \times 7$. A voltagem utilizada durante as observações variou de $15-25 \mathrm{kV}$, sempre em busca de se obter a melhor resolução. Depois de identificado, todo material foi depositado no Herbário do Departamento de Botânica da Universidade Federal de Santa Catarina (FLOR).

\section{Resultado e Discussão}

São apresentadas as descrições de três espécies representando as três famílias da ordem Corallinales. A família Sporolithaceae 
esteve representada pela espécie Sporolithon episporum, a família Hapalidiaceae pelo táxon Mesophyllum erubescens, enquanto a família Corallinaceae esteve representada pela espécie Lithophyllum stictaeforme. A seguir são apresentadas descrições dos gêneros e das espécies, além de comentários.

Sporolithaceae Verheij 1993: 195.

Sporolithon Heydrich 1897: 66.

A taxonomia de Sporolithon em nível específico é confusa, sendo que são raras as coleções onde as espécies destes táxons estejam determinadas. Embora alguns estudos tenham contribuído para melhorar o conceito de espécie no gênero (Verhij 1992; 1993), Dawson (1960) fornece uma chave geral para algumas espécies, baseando-se somente em caracteres como hábito e padrão de ramificação, que são considerados vagos ou ineficientes para a determinação das espécies. Keats \& Chamberlain (1993) realizaram estudos em duas espécies de Sporolithon, $S$. ptychoides e $S$. episporum, no qual forneceram, a partir da análise dos tipos, descrição detalhada e minuciosas ilustrações. Desde então, outros trabalhos têm sido realizados tornando mais preciso o conceito de espécie dentro deste gênero, dentre eles, Towsend et al. (1995), Alongi et al. (1996) e Harvey et al. (2002). Tomita (1976) descreveu oito espécies para o litoral brasileiro, propondo 5 novas combinações. A referida autora trabalhou com material dragado de toda a costa nacional, de profundidades que variaram de 16 a $116 \mathrm{~m}$.

O gênero é representado por crostas epígenas ou por nódulos lisos chegando a ramificados. Nas porções crostosas o talo é monômero e com organização radial nas porções protuberantes. Células de filamentos adjacentes unidas por conexões celulares secundárias e por fusões laterais. Tetrasporângios são cruciados e podem ocorrer isoladamente ou agrupando-se em soros separados por paráfises calcificadas.
Sporolithon episporum (M. Howe) E.Y. Dawson. Pacif. Nat. 1: 40. 1960.

Archeolithothamnion episporum Howe. US Nat. Mus. Bull.: 2. 1918.

Fig. 1 a-h

Talo crostoso com protuberâncias mameliformes, $2-5 \mathrm{~mm}$ diâm. Células epiteliais 3-4 $\mu \mathrm{m}$ diâm.; subepteliais variando de achatadas a alongadas, com 6-10 $\mu \mathrm{m}$ diâm. e 4-10 $\mu \mathrm{m}$ alt.; células do peritalo 5-10 $\mu \mathrm{m}$ diâm. e 9-15 $\mu \mathrm{m}$ alt.; hipotalo delgado com ca. $50 \mu \mathrm{m}$ de espessura com células 12-16 $\mu \mathrm{m}$ diâm. e 28-36 $\mu \mathrm{m}$ compr. (Figs. 1 a-c).

Tetrasporângios cruciados com 60-90 $\mu \mathrm{m}$ alt. e 30-40 $\mu \mathrm{m}$ diâm., dispostos em soros de tamanhos variados e de formato irregular, ligeiramente elevados em relação à superfície adjacente do talo vegetativo. Poros das câmaras com 9-15 $\mu$ m diâm. circundado por uma roseta com 12-14 células, freqüentemente alongadas radialmente. Câmara esporangial com 30-50 $\mu \mathrm{m}$ diâm. e 60-90 $\mu \mathrm{m}$ alt. Não foram observados soros imersos no talo, assim como talos gametofíticos.

Descrição e ilustrações adicionais: Keats \& Chamberlain (1993): 544-546, figs. 6-39 (exemplares tetraspóricos).

Material examinado: BRASIL. BAHIA: Cairú, Morro de São Paulo, 26.VIII.2000, J. M. C. Nunes \& A. Minervino Netto (FLOR 14507).

Distribuição no litoral brasileiro: Ceará (Tomita 1976) e Bahia (presente estudo).

Comentários: Espécie compondo rodolitos em bancos que ocorrem entre 10 a 20 metros profundidade.

Sporolithon episporum difere de $S$. ptychoides Heydrich pela presença de grandes poros esporangiais, ligações secundárias, raras fusões celulares, ausência de conceptáculos dispostos em linhas distintas. Na descrição do tipo de S. episporum, Howe (1918) observou que os esporângios são "imperfeitamente e irregularmente embebidos no talo". Nenhum exemplar do material examinado por Keats \& Chamberlain (1993) apresentavam esporângios imersos, e na espécie tipo também não foram 

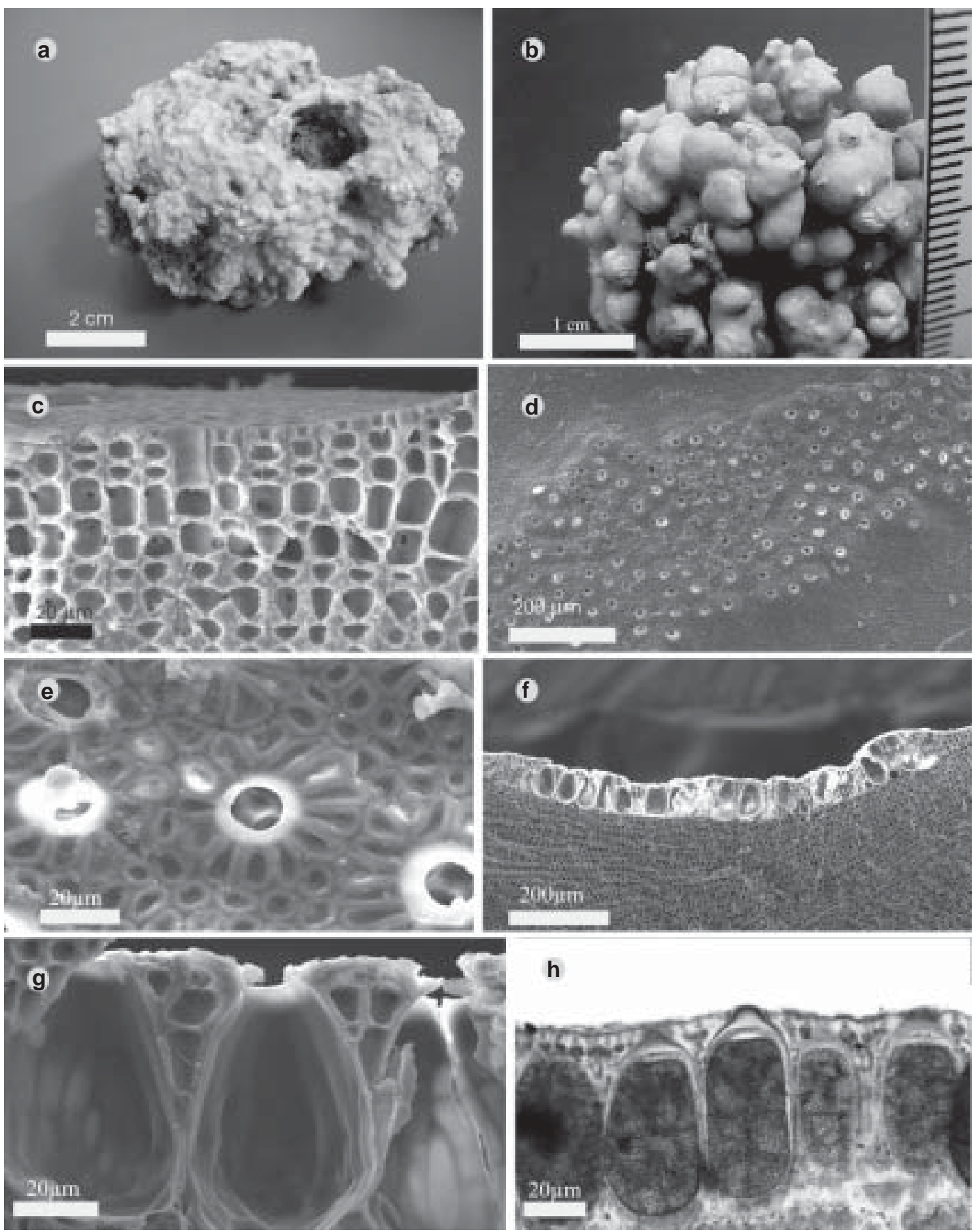

h

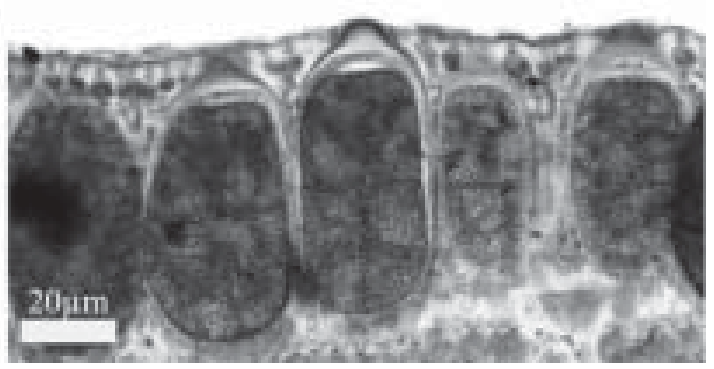

Figura 1 - a-h. Aspecto geral e micrografias de Sporolithon episporum. a, b. Diferentes aspectos de rodolitos formados pela referida espécie. c. Fratura transversal da crosta evidenciando células epiteliais em forma de taça, fusões e conexões celulares secundárias unindo filamentos adjacentes. d. Vista superficial de um soro tetrasporangial. e. Detalhe da vista superficial de um soro evidenciando a disposição em roseta das células que delimitam os poros (ponta de seta). f. Fratura transversal a um soro tetrasporangial evidenciando sua presença na superfície do rodolito. g. Detalhe das câmaras esporangiais. h. Corte transversal de um soro evidenciando os tetrasporângios cruciados (setas). 
observados esporângios imersos no talo. A combinação principal de ligações secundárias, ausência de esporângios imersos, sempre com formação do esporângio entre uma camada de células alongadas, e soro esporangial elevado são características diagnósticas desta espécie (Keats \& Chamberlain 1993).

Estão referidas para o Brasil oito espécies de Sporolithon, mas nenhuma delas foi revisada segundo as técnicas e nomenclatura moderna (Horta 2002). Considerando a característica diagnóstica utilizada por Tomita (1976) e por autores mais recentes (Keats \& Chamberlain 1993; Verheij 1993), a ausência de soros enterrados no talo representa uma importante informação, que, em conjunto com coincidências morfométricas, corroboram a presente identificação.

Hapalidiaceae J. E. Gray 1864: 22.

Mesophyllum Lemoine 1928: 251.

O gênero Mesophyllum foi estabelecido por Lemoine (1928) para espécies melobesoideas com medula coaxial. Woelkerling \& Harvey $(1992,1993)$ questionaram a validade desta característica para a delimitação genérica porque algumas espécies combinam áreas coaxiais e não-coaxiais em alguns talos. Ao invés disso Woelkerling \& Harvey $(1992,1993)$ delimitaram Mesophyllum com base na anatomia dos conceptáculos espermatangiais, como sugerido por Suneson (1937), Lebednik (1978), Townsend (1979), Woelkerling \& Harvey (1992), Keats \& Maneveldt (1997) e Athanasiadis et al. (2004), incluindo outros caracteres como: 1 . construção monômera; 2. haustório ausente; 3 . paredes externas das células epiteliais esféricas ou achatadas, mas nunca em forma de taça; 4 . ativa divisão das células sub-epiteliais, usualmente alongadas; 5. células mãe dos espermatângios não ramificadas ocorrendo tanto no teto quanto no assoalho do conceptáculo; 6. câmaras dos conceptáculos carposporangiais com a forma de halteres.
Mesophyllum erubescens (Foslie) Lemoine Bull. Soc. Bot. Fr. 75: 252. 1928.

Lithothamnion erubescens Foslie Norsk Vid. Selsk. Skr. 5: 9-10. 1900.

Fig. 2 a-h

Talo não articulado, fruticoso, com protuberâncias medindo até $20 \mathrm{~mm}$ alt. e 25 mm diâm., com ramificação sub-dicotômica, cor rosa claro. Porção crostosa de organização monômera medindo 0,9-1,7 mm de espessura. Medula coaxial com apenas uma camada de células epiteliais de forma esférica com aproximadamente $5 \mu \mathrm{m}$ alt. e 5-8 $\mu \mathrm{m}$ diâm.; células subepiteliais alongadas com 7-16 $\mu \mathrm{m}$ alt. e 4-8 $\mu \mathrm{m}$ diâm.; células de filamentos adjacentes unidas por fusões celulares; conexões celulares secundárias não foram observadas.

Conceptáculos tetrasporangiais multiporados elevados em relação à superfície do talo adjacente, com câmaras medindo de $195-310 \mu \mathrm{m}$ diâm. e até $150 \mu \mathrm{m}$ alt.; teto com 4-5 camadas de células de espessura; piso do conceptáculo 10 camadas de células abaixo da superfície do talo; poros com 5-12 $\mu \mathrm{m}$ diâm. e aproximadamente $30 \mu \mathrm{m}$ alt., localizados em depressões; células basais dos filamentos que delimitam o canal dos poros alongadas com 3-5 $\mu \mathrm{m}$ diâm. e 10-15 $\mu \mathrm{m}$ alt. Tetrasporângios com 65-80 $\mu \mathrm{m}$ diâm. e 90 a $110 \mu \mathrm{m}$ alt. Conceptáculos espermatangiais uniporados com câmara com 80-148 $\mu \mathrm{m}$ diâm. e 55-72 $\mu \mathrm{m}$ alt., com teto composto por 5-7 camadas de células e piso com até 15 camadas de células abaixo da superfície do talo. Conceptáculos carposporangiais com câmara em forma de halteres diâmetro variando de 230-310 $\mu \mathrm{m}$ e altura de 110-205 $\mu \mathrm{m}$. Carpósporos com 70-135 $\mu \mathrm{m}$ diâm.

Material examinado: BRASIL. BAHIA: Prado, Cumuruxatiba, 10.III.2001, J. M. C. Nunes \& G. M. Lyra, (FLOR 14510, 14511).

Distribuição no litoral brasileiro: Santa Catarina (Horta et al. 2007), Bahia (Figueiredo $\&$ Steneck 2002) e Fernando de Noronha (Foslie 1900). 

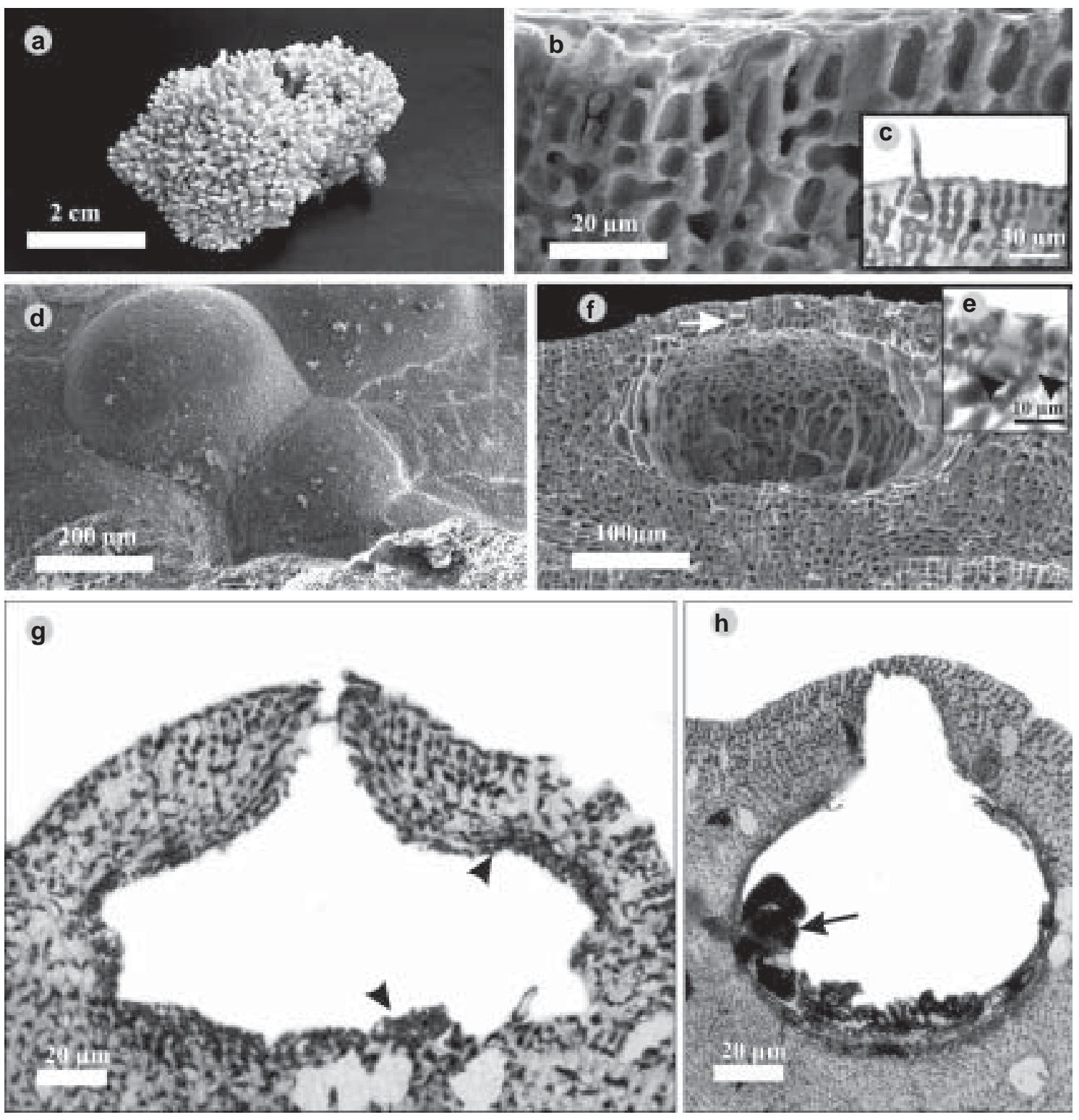

Figura 2 - a-h. Aspecto geral e micrografias de Mesophyllum erubescens. a. Aspectos geral de crosta protuberante crescendo sobre fragmento de recife. b. Fratura transversal da crosta evidenciando células sub-epiteliais alongadas e fusões celulares secundárias unindo filamentos adjacentes. c. Corte transversal da região epitelial destacando-se a presença de tricocito. d. Vista frontal de um conceptáculo tetrasporangial multiporado. e. Secção transversal de um conceptáculo tetrasporangial evidenciando-se poro (seta). f. Detalhe das células basais que delimitam o canal do poro alongadas longitudinalmente (pontas de seta). g. Corte transversal de um conceptáculo espermatangial uniporado, destacando a presença de espermácios tanto no teto quanto no assoalho da câmara (pontas de setas). h. Corte transversal de um conceptáculo carposporangial com a presença de carpósporos (seta). 
Comentários: crescendo no mesolitoral, epilítica na região frontal da formação recifal (RFR).

Figueiredo \& Steneck (2002) faz referência deste táxon como sendo a primeira para o litoral brasileiro, contudo Foslie (1900) baseia a descrição do gênero em material oriundo da costa brasileira, identificando o mesmo como Lithothamniom erubescens.

Woelkerling \& Harvey (1992) questionaram a validade de muitas das características tradicionalmente usadas para a distinção entre as espécies de Mesophyllum, e notou que caracteres associados com o teto do conceptáculo esporangial parecem ser estáveis e úteis para a distinção entre as espécies de Mesophyllum na Austrália. A estrutura do poro foi particularmente significante. A estrutura do poro do conceptáculo em material coletado recentemente de $M$. erubescens foi idêntica a estrutura do poro dos espécimes tipo de $M$. erubescens e M. madagascariensis, e com M. erubescens documentado por Verheij (1993).

Keats \& Chamberlain (1994) aplicaram o epíteto erubescens para o material por eles estudado por este ter prioridade histórica sobre madagascariensis. Estes autores consideraram como caracteres diagnósticos: a. conceptáculos tetrasporangiais e bisporangiais elevados, sem margem elevada; b. poros tetrasporangiais e bisporangiais circundados por células em rosetas localizados em depressão em relação à superfície adjacente; c. canal dos poros de conceptáculos tetrasporangiais e bisporangiais, composto por filamentos de células que possuem diâmetro semelhante ao das células que compõem o teto, diferenciam-se das demais por apresentarem célula basal do filamento alongada.

Os exemplares estudados apresentaram características anatômicas e morfológicas referentes ao gênero Mesophyllum. De acordo com as observações foi possível determinar a espécie devido a: forma das células basais dos filamentos que delimitam o canal do poro do conceptáculo tetrasporangial, o número de poros no teto do conceptáculo maior que 50 e teto do conceptáculo entre 4 a 6 camadas de células distante da superfície do talo (Tab. 1).

Corallinaceae Lamouroux 1812: 185.

Lithophyllum Phil. 1837: 387.

Philippi (1837) estabeleceu o gênero Lithophyllum para as plantas calcárias rígidas consistindo de expansões foliares. Previamente organismos com esta descrição foram incluídos entre os nulipores, e estes classificados como animais representantes dos hidrocorais. Baseado em coleções da costa da Sicília, Philippi (1837) determinou quatro espécies, três das quais (L. expansum, $L$. incrustans, $L$. lichenoides) foram recentemente descritas. A quarta espécie (L. decussatum), Philippi listou Millepora decussata Ellis \& Solander como sinônimo (Woelkerling 1983).

O Gênero Lithophyllum é caracterizado por apresentar conceptáculos tetrasporangiais uniporados, células de filamentos adjacentes unidas por conexões celulares secundárias e margem de crescimento polistromática de organização dímera ou monômera (Woelkerling 1996).

Lithophyllum stictaeforme (Areschoug in J. Agardh) Hauk. Oesterr. Bot. Zeitschr 27: 292. 1877.

Fig. 3 a-f

Melobesia stictaeformis Arechoug in $\mathrm{J}$. Agardh. Sp. Gen. Ord. Algar. II: 517. 1852.

Talo protuberante crescendo sobre formações recifais. Protuberâncias com até $40 \mathrm{~mm}$ compr. e 5-8 mm diâm. Talo de organização dímera, com margem poliestromática, conexões celulares secundárias unindo células de filamentos adjacentes. Células epiteliais de 1-2 camadas, com 5-9 $\mu \mathrm{m}$ diâm. e 2-3 $\mu \mathrm{m}$ alt. Conceptáculos tetrasporangiais uniporados, columela na porção central, câmara com 202$262 \mu \mathrm{m}$ diâm. e 105 a $182 \mu \mathrm{m}$ alt.; teto do conceptáculo tetrasporangial no mesmo plano da superfície adjacente do talo, com 5 a 7 células de espessura. Tetrasporângios com 15$33 \mu \mathrm{m}$ diâm. e 46-68 $\mu \mathrm{m}$ alt. 

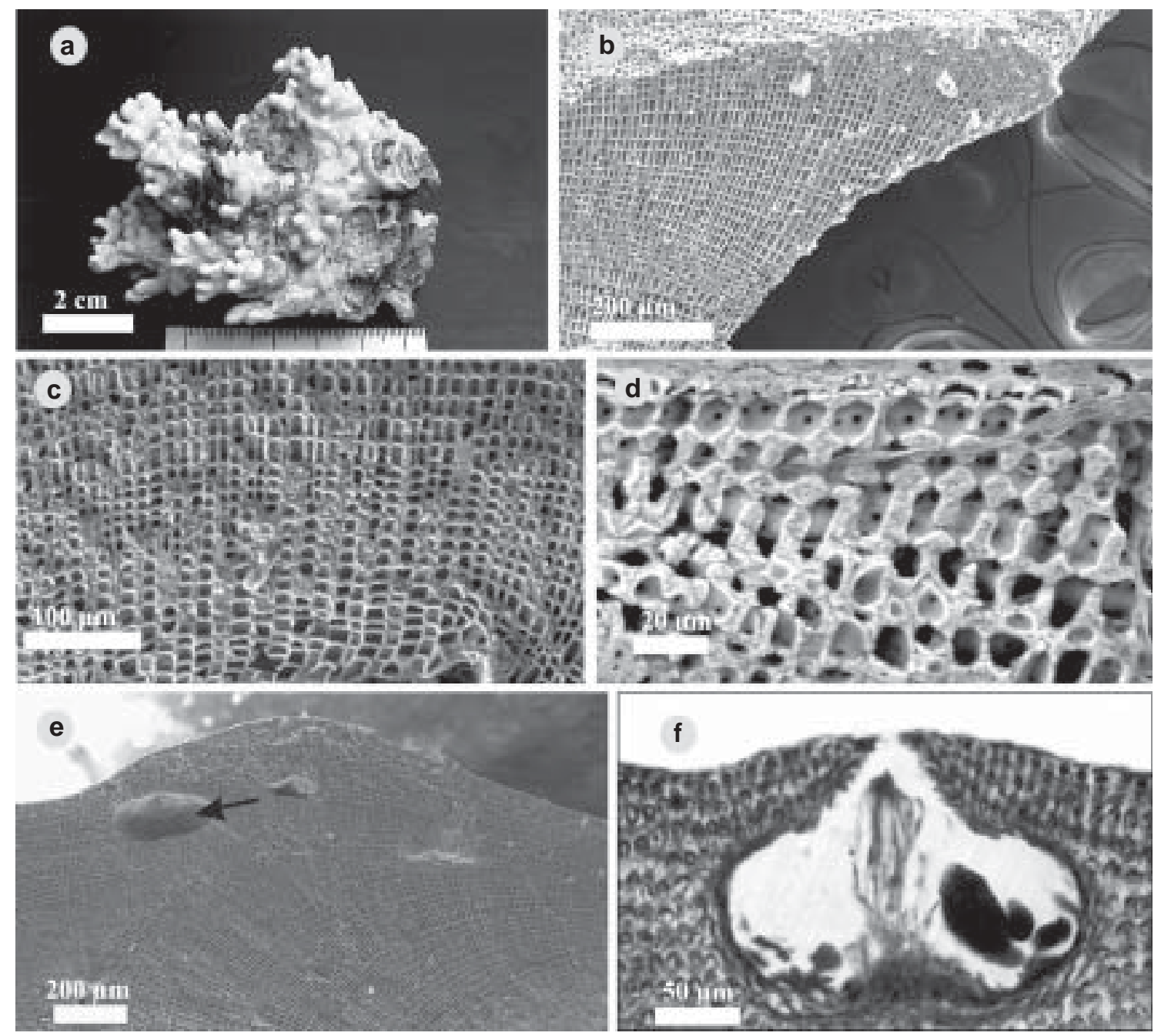

Figura 3 - a-f. Aspecto geral e micrografias de Lithophyllum stictaeforme. a. Aspecto geral de crosta protuberante crescendo sobre fragmento de recife. b. Fratura transversal da margem de crescimento polistromática de organização dímera. c. Detalhe de uma fratura transversal ilustrando organização dímera com destaque (seta) para as células quadráticas do hipotalo. d. Detalhe de uma fratura transversal ilustrando a disposição das células epiteliais (seta). e. Fratura transversal de uma porção do talo com conceptáculos tetrasporangiais enterrados. f. Corte transversal de um conceptáculo tetrasporangial.

Descrição e ilustrações adicionais: Athanasiadis (1999): 737, figs. 1-12 (exemplares masculinos).

Material examinado: BRASIL. BAHIA: Prado, Cumuruxatiba, 10.III.2001, J. M. C. Nunes \& G. M. Lyra (FLOR 14512).

Distribuição no litoral brasileiro: Bahia (presente estudo).

Comentários: crescendo no mesolitoral, epilítica na região frontal do recife (RFR).
Melobesia stictaeformis foi originalmente descrita por Areschoug (in J. Agardh 1852, p. 517) com base em material proveniente de coleções oriundas do Mediterrâneo com o nome de Millepora coriacea Linné (1767, p. 1285). Hauck (1877) transferiu a espécie para Lithophyllum.

Lithophyllum congestum (Foslie) Foslie, listado para a região de Abrolhos, região sul do litoral baiano, por Figueiredo \& Steneck 


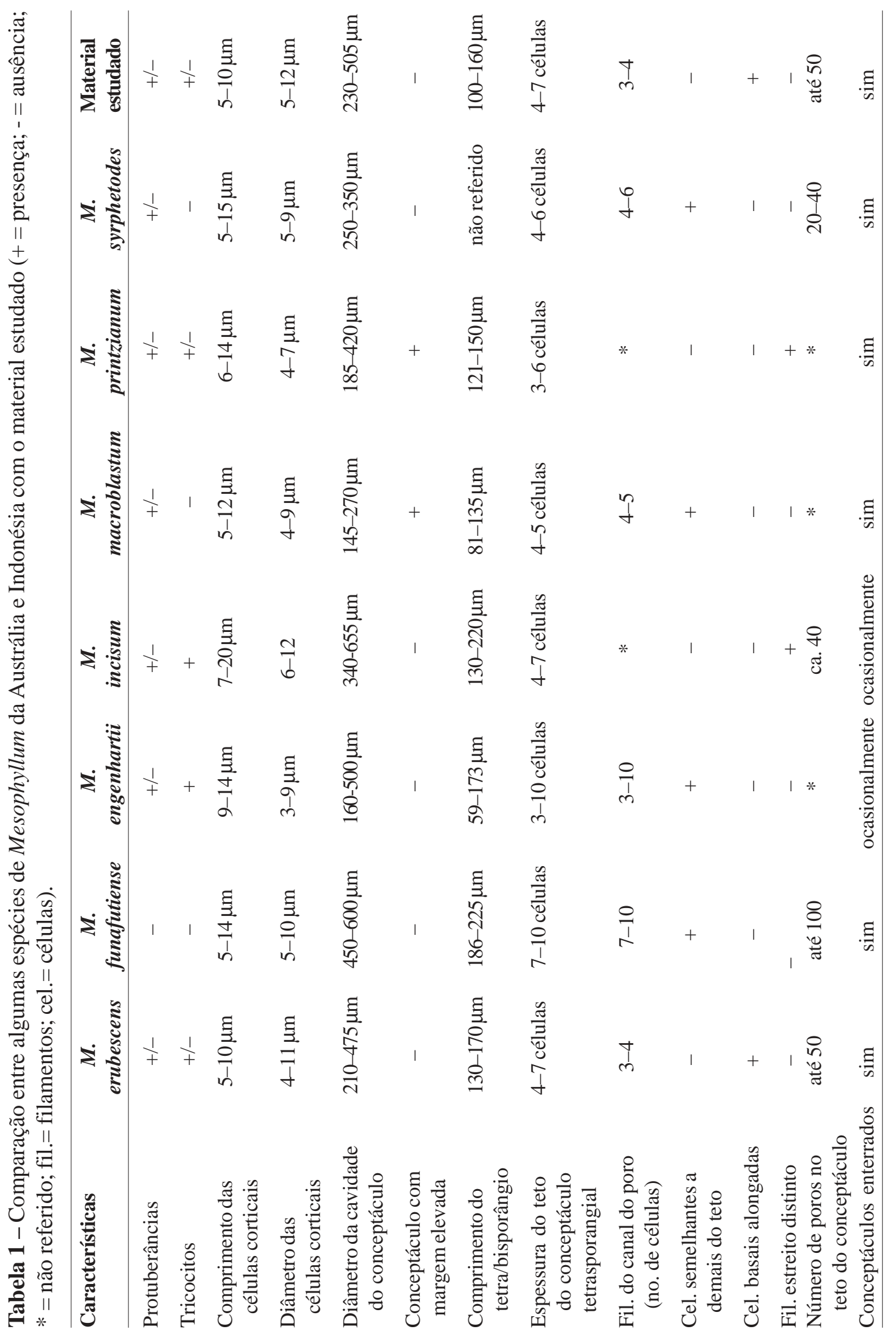

Rodriguésia 59 (1): 075-086. 2008 
(2002), apresenta conceptáculos tetrasporangiais com o diâmetro variando de 216-421 $\mu \mathrm{m}$ (Littler \& Littler 2000), portanto, maiores que os observados nos espécimes por nós analisados. Entretanto, para que uma análise mais criteriosa seja feita, a referida espécie precisa ser descrita mais detalhadamente com o uso de técnicas modernas.

\section{Agradecimentos}

Ao PICDT - CAPES pela concessão da bolsa de Pós-Graduação, Dra. Zenilda Bouzon do Departamento de Biologia Celular da Universidade de Santa Catarina pelo uso irrestrito das dependências do Laboratório de Algas Marinhas.

\section{REFERÊNCIAS BIBLIOGRÁFICAS}

Agardh, J. 1852. Species Genera et Ordinales Algarum 2(2): 337-720.

Alongi, G.; Cormaci, M. \& Furnari, G. 1996. On the occurrence of Sporolithon ptychoides Heydrich (Corallinales, Sporolithaceae, Rhodophyta) in the Mediterranean Sea. Cryptogamie, Algologie 17: 131-137.

Athanasiadis, A. 1999. The taxonomic status of Lithophyllum stictaeforme (Rhodophyta, Corallinales) and its generic position in light of phylogenetic considerations. Nordic Journal of Botany 19: 735-745.

Athanasiadis, A.; Lebednik, P. \& Adey, W. H. 2004. The genus Mesophyllum (Melobesioideae, Corallinales, Rhodophyta) on the northern Pacific coast of North America. Phycologia 43: 126-165.

Dawson, E. Y. 1960. New records of marine algae from Pacific Mexico and Central America. Pacific Naturalist 1: 31-52.

Figueiredo, M. A. O. 1997. Colonization and growth of crustose coralline algae in Abrolhos, Brazil. Proceedings of the 8th International Coral Reef Symposium 1: 689-693.

Figueiredo, M. A. O. \& Steneck, R. S. 2002. Floristic and ecological studies of crustose coralline algae on Brazil's Abrolhos reefs. 9th International Coral Reef Symposium 1: 493-498.

Foslie, M. 1900. Revised systematical survey of the Melobesiea. Kongelige Norske Vindenskabers Selskabs Skrifter 5: 1-22.

Harvey, A. S.; Woelkerling, W. J. \& Millar, A. J. K. 2002. The Sporolithaceae (Corallinales, Rhodophyta) in southeastern Australia: taxonomy and 18S rRNA phylogeny. Phycologia 41: 207-227.

Harvey, A. S.; Broadwater, S. T.; Woelkerling, W. J. \& Mitrovski, P. J. 2003. Choreonema (Corallinales, Rhodophyta): 18S rDNA phylogeny and resurrection of the Hapalidiaceae for the subfamilies Choreonematoideae, Austrolithoideae and Melobesioideae. Journal of Phycology 39: 988-998.

Hauck, F. 1877. Verzeichness der im Golfe von Trieste gesammelten Meeralgen. 2. Nachtrag. Österreichische Botanische Zeitschrift 27: 50.

Horta, P. A.; Scherner, F.; Riosmena, R.; Bouzon, Zenilda, L. 2007. Morphology and Reproduction of Mesophyllum erubescens - a redescription of an old Brazilian coralline name. Revista Brasileira de Botânica. no prelo.

Horta, P. A. 2002. Bases para a identificação das coralináceas não articuladas do litoral brasileiro - uma síntese do conhecimento. Biotemas 15: 7-44.

2000. Macroalgas do infralitoral do sul e sudeste do Brasil: Taxonomia e Biogeografia. Tese de Doutorado. Universidade de São Paulo, São Paulo, $301 \mathrm{p}$.

Howe, M. A. 1918. On some fossil and recent Lithothamnieae of the Panama Canal Zone. Smithsonian Institution, United States National Museum Bulletin 103: 1-13.

Keats, D. W. \& Chamberlain, Y. M. 1994. Two melobesioid coralline algae (Rhodophyta, Corallinales): Mesophyllum erubescens (Foslie) Lemoine and Mesophyllum funafutiense (Foslie) Verheij from 
Sodwana Bay, South Africa. South African Journal of Botany 60: 175-190

1993. Sporolithon ptychoides Heydrich and S. episporum (Howe) Dawson: two crustose coralline red algae (Corallinales, Sporolithaceae) in South Africa. South African Journal of Botany 59: 541-550.

Keats, D. W. \& Maneveldt, G. 1997. First report of the melobesioid alga (Corallinales, Rhodophyta) Mesophyllum incisum (Foslie) Adey in South Africa. South African Journal of Botany 63 (4): 201-209.

Lebednik, P. A. 1978. Development of male conceptacles in Mesophyllum Lemoine and other genera of the Corallinaceae (Rhodophyta). Phycologia 17: 388-395.

Lemoine, M. 1928. Un nouveau genre de Mélobésiées: Mesophyllum. Bulletin de la Societe Botanique de France 75: 251-254.

Linné, V. C. 1767. Systema naturae 2: 1-73.

Littler, M. M. \& Littler, D. S. 2000. Caribbean reef plants. Off Shore Graphics, Washington, D.C.

Martens, G von. 1870. Conspectus algarum Brasiliae hactenus detectarum. Vidensk Meddr dansk naturh. Foren 2:297-314.

Moura, C. W. N.; Kraus, J. E. \& CordeiroMarinho, M. 1997. Metodologia para obtenção de cortes histológicos com historresina e coloração com azul de toluidina $O$ para algas coralináceas (rhodophyta Corallinales). Hoehnea 24: 17-27.

Oliveira-Filho, E. C.; Ugadim, Y. \& de Paula, É. J. 1979. Associated epibiota on Sargassum floating on the waters of the Brazilian current - biogeographical remarks. Boletim de Botânica da Universidade de São Paulo 7: 5-9.

Philippi, R. A. 1837. Beweis, dass die Nulliporen Pflanzen sind. Archiv Für Naturgeschichte 3: 387-393.

Riosmena-Rodriguez, R. 1993. Una Propuesta de tecnica histológica para el estudio de algas coralinas (Corallinales: Rhodophyta). Revista Investigación Científica 4: 65-73.
Suneson, S. 1937. Studien über die Entwicklungsgeschichte der Corallinaceen. Lunds Universitets Årsskrift, Ny Följd, Andra Afdelningen 2, 33(2): 1-101.

Tâmega, F. T. S. \& Figueiredo, M. A. O. 2005. Distribuição das algas calcárias incrustantes (Corallinales, Rhodophyta) em diferentes habitats na Praia do Forno, Armação dos Búzios, Rio de Janeiro. Rodriguésia 56: 123-132.

Taylor, W. R. 1931. A synopsis of the marine algae of Brazil. Revue Algologique 5: 279-313.

Tomita, N. Y. 1976. Contribuição ao conhecimento do gênero Sporolithon (Corallinaceae, Cryptonemiales) no Brasil. Tese de Doutorado. Universidade de São Paulo, São Paulo, 138p.

Townsend, R. A. 1979. Synarthrophyton, a new genus of Coralinaceae (Cryptonemiales, Rhodophyta) from the southern hemisphere. Journal of Phycology 15: 251-259.

Townsend, R. A.; Woelkerling, W. J.; Harvey, A. S. \& Borowitzka, M. 1995. An account of the red algal genus Sporolithon (Sporolithaceae, Corallinales) in southern Australia. Australian Systematic Botany 8: 85-121.

Towsend, R. A. \& Borowitzka, M.A. 2001. Heydrichia homalopasta sp nov. (Sporolithaceae, Rhodophyta) from Australia. Botanica Marina 44: 237-244.

Verheij, E. 1993. The genus Sporolithon (Sporolithaceae, fam. nov., Corallinales, Rhodophyta) from the Spermond Archipelago, Indonesia. Phycologia 32: 184-196.

Verheij, E. 1992. Structure and reproduction of Sporolithon episoredion (Adey, Townsend et Boykins) comb. nov. (Corallinales, Rhodophyta) from the Spermonde Archipelago, Indonesia. Phycologia 31: 500-509.

Woelkerling, W. J. \& Harvey, A. 1993. An account of southem Australia species Mesophyllum (Corallinaceae, Rhodophyta). Australian Journal of Systematic Botany 6: 571-637. 
1992. Mesophyllum incisum (Corallinaceae, Rhodophyta) in southem Australia: implications for generic and specific delimitation in the Melobesioideae. British Phycology Journal 27: 381-399.

Woelkerling, W. J. 1996. The order Corallinales. In: Womersley, H. B. S. (ed.). The Marine Benthic Flora of Sothern Australia, Part IIIB. Graphic Print Group, Adelaide. Pp. 146-323.
1988. The Coralline red algae: An analysis of the genera and subfamilies of nongeniculate corallinaceae. Oxford University Press, Oxford.

1983. A taxonomic reassessment of Lithophyllum (Corallinaceae, Rhodophyta) based on studies of R. A. Philippi's original collections. British Phycology Journal 18: 299-328. 